\title{
Identification of identifiable parameters affecting aerodynamic performance
}

\author{
R.Safarov ${ }^{1}$, A.R.Safarov ${ }^{2}$, \\ Samarkand State Institute of Architecture and Civil Engineering \\ safarov-raxmon@mail.ru, safarov-akbar@mail.ru
}

\begin{abstract}
The developed scientific works are directly related to solving the problems of identifying multi-parameter objects. The proposed algorithm is effectively implemented on a computer, which is confirmed by calculations of problems of supersonic aerodynamics.
\end{abstract}

Keywords- algorithm, identification, object, supersonic, aerodynamics.

\section{INTRODUCTION}

Let us consider the methods of identification and assessment of the state in solving problems of the process of flowing around an oscillating profile by a supersonic flow.

Using the proposed algorithm, we obtain identification models. As an aerodynamic characteristic used shock wave departure $\Delta^{-}$. The main factors influencing the response function are: X_1-Mach number $\mathrm{M} \_\infty, \mathrm{X} \_$2-relative distance from the bottom edge of the profile to the screen $\mathrm{H}^{-}, \mathrm{X} \_$-angle of attack of the profile $\alpha, X_{-}$4-relative thickness of the profile $\mathrm{C}$. The process of building the model consists of three stages. At the first stage, the linear part of the model is considered. The second is the quadratic part and the third is the nonlinear part.

To achieve the resulting conditioning of the matrices, the experiments were conducted by the method of rotationally central compositional planning.

At the first stage, the linear model has the form

$$
\mathrm{y}=a_{0}+\sum_{i=1}^{4} a_{i} X_{i}
$$

On the first row of selection we get six combinations (of four in two) linear polynomials. The private polynomial can be written as:

$$
\begin{aligned}
& \mathrm{y}_{1 i}=a_{0 i}+a_{1 i} X_{1}+a_{2 i} X_{i+1}, i=\overline{1,3}, \\
& \mathrm{y}_{2 i+2}=a_{0} i+3+a_{1 i+3} X_{2}+a_{2 i+3} X_{i+2}, i=\overline{1,2}, \\
& \mathrm{y}_{34}=a_{06}+a_{16} X_{3}+a_{26} X_{4} .
\end{aligned}
$$

To determine the coefficients a_0i, a_1i, a_2i, $(i=(1,6) \overline{)}$ we compose a system of conditional equations in the form

$$
\left\{\begin{array}{c}
\sum_{k=1}^{N} \mathrm{y}_{i j}^{k}-a_{0} N-a_{i} \sum_{k=1}^{N} X_{i k}-a_{j} \sum_{k=1}^{N} X_{j k}=0 \\
\sum_{k=1}^{N} \mathrm{y}_{i j}^{k} X_{i k}-a_{0} \sum_{k=1}^{N} X_{i k}-a_{i} \sum_{k=1}^{N} X_{i k}^{2}-a_{j} \sum_{k=1}^{N} X_{j k} X_{i k}=0 \\
\sum_{k=1}^{N} \mathrm{y}_{i j}^{k} X_{j k}-a_{0} \sum_{k=1}^{N} X_{j k}-a_{i} \sum_{k=1}^{N} X_{i k} X_{j k}-a_{j} \sum_{k=1}^{N} X_{j k}^{2}=0
\end{array}\right.
$$

where $i=\overline{1, n}, j=\overline{l+1, n}$.

The system of equations is solved by the Gauss method. The standard error is calculated by the formula

$$
\delta_{s}^{2}=\frac{1}{N} \sum_{k=1}^{N}\left(\mathrm{y}_{i j k}^{\ni}-\mathrm{y}_{i j k}^{\mathrm{p}}\right)^{2}, s=\overline{1, \mathrm{C}_{n}^{2}}
$$

where $\mathrm{Y}_{i j k}^{\mathrm{g}}$-experimental values at the i-th point; ${ }_{i j k}^{\mathrm{p}}$ - estimated value of private descriptions; $\delta_{s}^{2}-$ standard error of the sequence of models.

\section{MAIN PART}

We will gradually increase the complexity of the partial and complete regression equations and at the same time trace the nature of the change in the mean square error.

The first stage of building the model - on the first row of selection, the following coefficients were obtained, and accordingly, the standard deviations (Table 1).

Table. 1. 


\begin{tabular}{|c|c|c|c|c|}
\hline $\mathrm{I}$ & $\boldsymbol{a}_{\mathbf{0 i}}$ & $\boldsymbol{a}_{\mathbf{1 i}}$ & $\boldsymbol{a}_{\mathbf{2 i}}$ & $\boldsymbol{\delta}_{\boldsymbol{i}}^{\mathbf{2}}$ \\
\hline 1 & 1.6316 & -0.3005 & 0.0847 & 0.4017 \\
\hline 2 & 1.7807 & -0.0256 & -0.4017 & 0.5443 \\
\hline 3 & 1.5347 & -0.4791 & 0.0654 & 0.4358 \\
\hline 4 & 1.5757 & 0.0723 & -0.5428 & 0.4367 \\
\hline 5 & 1.4079 & 0.0353 & 0.0166 & 0.5496 \\
\hline 6 & 1.4788 & -0.6092 & 0.0439 & 0.4181 \\
\hline
\end{tabular}

In the next series of selection, those variables that satisfy the condition

$$
\delta_{i}^{2}<\delta_{\mathrm{cp},}^{2} i=\overline{1,6}
$$

Among the obtained variables satisfying the criterion U_14, U_23, U_34.

In the second row of selection, we obtained with a combination (of three by two) linear polynomials

$$
\begin{gathered}
Z_{14-23}=b_{01}+b_{11} \mathrm{y}_{14}+b_{21} \mathrm{y}_{23}, Z_{14-34}=b_{02}+b_{12} \mathrm{y}_{14}+b_{22} \mathrm{y}_{34}, \\
Z_{23-34}=b_{03}+b_{13} \mathrm{y}_{23}+b_{23} \mathrm{y}_{34} .
\end{gathered}
$$

The numerical values of the table 2 are obtained.

Table.

2.

\begin{tabular}{|c|c|c|c|c|}
\hline $\mathrm{I}$ & $\boldsymbol{b}_{\mathbf{0} \boldsymbol{i}}$ & $\boldsymbol{b}_{\mathbf{1 i}}$ & $\boldsymbol{b}_{\mathbf{2 i}}$ & $\boldsymbol{\delta}_{\boldsymbol{i}}^{\mathbf{2}}$ \\
\hline 1 & -1.5231 & 0.9629 & 0.9626 & 0.2621 \\
\hline 2 & -0.7688 & 0.7151 & 0.7521 & 0.3343 \\
\hline 3 & -0.2774 & 0.5173 & 0.6512 & 0.3931 \\
\hline
\end{tabular}

From the obtained results it is seen that only $Z_{-}$(14-23) satisfy criterion (2).

Then $\quad Z_{14-23}=b_{01}+b_{11} \mathrm{y}_{14}+b_{21} \mathrm{y}_{23}$

$$
\begin{gathered}
\mathrm{y}_{14}=a_{03}+a_{13} \mathrm{X}_{1}+a_{23} \mathrm{X}_{4}, \mathrm{y}_{23}=a_{04}+a_{14} \mathrm{X}_{2}+a_{24} \mathrm{X}_{3} \\
Z_{14-23}=b_{01}+b_{11} a_{03}+b_{21} a_{04}+b_{11} a_{13} \mathrm{X}_{1}+b_{21} a_{14} \mathrm{X}_{2}+b_{21} a_{24} \mathrm{X}_{3}+b_{11} a_{23} \mathrm{X}_{4} \\
a_{0}=b_{01}+b_{11} a_{03}+b_{21} a_{04}=1.4714, \quad a_{1}=b_{11} a_{13}=-0.4614 \\
a_{2}=b_{21} a_{14}=0.0696, a_{3}=b_{21} a_{24}=-0.5224, a_{4}=0.063
\end{gathered}
$$

At the first stage, a linear model is obtained.

$$
\mathrm{y}=1.471-0.4614 \mathrm{X}_{1}+0.0696 \mathrm{X}_{2}-0.5224 \mathrm{X}_{3}+0.063 \mathrm{X}_{4} \text {. }
$$

The second stage of building the model.

In the first row of selection, the following coefficients were obtained, respectively, with standard deviations (Table 3).

Table 3.

\begin{tabular}{|l|l|l|l|l|}
\hline $\mathrm{I}$ & $\boldsymbol{a}_{\mathbf{0} \boldsymbol{i}}^{\prime}$ & $\boldsymbol{a}_{\mathbf{1 i}}^{\prime}$ & $\boldsymbol{a}_{\mathbf{2} \boldsymbol{i}}^{\prime}$ & $\boldsymbol{\delta}_{\boldsymbol{i}}^{\mathbf{2}}$ \\
\hline 1 & 0.1232 & $-0,0622$ & -0.0005 & 0.2443 \\
\hline 2 & 0.0951 & -0.0653 & 0.0343 & 0.2436 \\
\hline 3 & 0.136 & -0.0547 & -0.0003 & 0.2433 \\
\hline 4 & 0.017 & -0.0013 & 0.0424 & 0.2559 \\
\hline 5 & 0.0833 & -0.0006 & -0.0005 & 0.2512 \\
\hline 6 & 0.0511 & 0.0428 & -0.0006 & 0.2501 \\
\hline
\end{tabular}

$\delta_{\mathrm{cp}}^{2}=0.2479$

In the second row of selection (table. 4.)

Table. 4.

\begin{tabular}{|l|l|l|l|c|}
\hline $\mathrm{I}$ & $\boldsymbol{b}_{\mathbf{0} \boldsymbol{i}}^{\prime}$ & $\boldsymbol{b}_{\mathbf{1} \boldsymbol{i}}^{\prime}$ & $\boldsymbol{b}_{\mathbf{2} \boldsymbol{i}}^{\prime}$ & $\boldsymbol{\delta}_{\boldsymbol{i}}^{\mathbf{2}}$ \\
\hline 1 & 0 & 0.3228 & 0.6991 & 0.2434 \\
\hline 2 & 0 & 0.0825 & 0.9265 & 0.2423 \\
\hline 3 & 0 & 0.3647 & 0.6831 & 0.2418 \\
\hline
\end{tabular}

$\delta_{\mathrm{cp}}^{2}=0.2425$ 
In the third row of selection

$$
\begin{gathered}
\mathrm{c}_{01}=0, \quad \mathrm{c}_{11}=-0.1206, \quad \mathrm{c}_{12}=1.1182, \quad \delta^{2}=0.2418 \\
v_{1214-1324}=c_{01}+c_{11} z_{12-14}+c_{21} z_{13-14},{ }^{\prime} \\
z_{12-14}=b_{02+}^{\prime} b_{12}^{\prime} \mathrm{y}_{12}+b_{22}^{\prime} \mathrm{y}_{14}, z_{13-14}=b_{03}^{\prime}+b_{13}^{\prime} \mathrm{y}_{13}+b_{23}^{\prime} \mathrm{y}_{14} \\
\mathrm{y}_{1 i+1}=a_{0 i}^{\prime}+a_{1 i}^{\prime} \widetilde{\mathrm{X}}_{1}+a_{2 i}^{\prime} \widetilde{\mathrm{X}}_{i+1}, i=\overline{1,3} \\
v_{1214-1314}=c_{11} b_{12} a_{01}+c_{11} b_{22} a_{03}+c_{21} b_{13} a_{02}+\left(c_{11} b_{12} a_{11}+c_{11} b_{22} a_{13}+\right. \\
\left.c_{21} b_{13} a_{13}+c_{21} b_{23} a_{13}\right) \widetilde{\mathrm{X}}_{1}+\left(c_{11} b_{12} a_{21}+c_{11} b_{22} a_{23}\right) \widetilde{\mathrm{X}}_{2}+c_{21} b_{13} a_{23} \widetilde{\mathrm{X}}_{3}+ \\
c_{21} b_{23} a_{23} \widetilde{\mathrm{X}}_{4}
\end{gathered}
$$

At the second stage, the model is obtained

$$
\widetilde{\mathrm{y}}_{0}^{\prime}=0.0224-0.0617 \mathrm{X}_{1}^{2}+0.014 \mathrm{X}_{2}^{2}-0.0002 \mathrm{X}_{4}^{2}, \delta^{2}=0.2418
$$

The third stage of building the model.

In the first row of selection we obtain the following coefficients, respectively, with standard deviations (Table 5).

Table. 5 .

\begin{tabular}{|c|l|l|l|}
\hline $\mathrm{I}$ & $\boldsymbol{a}_{\mathbf{0} \boldsymbol{i}}{ }^{\prime \prime}$ & $\boldsymbol{a}_{\mathbf{1} \boldsymbol{i}}$ & $\boldsymbol{\delta}_{\boldsymbol{i}}^{\mathbf{2}}$ \\
\hline 1 & 0.0313 & -0.0063 & 0.2392 \\
\hline 2 & 0.0462 & -0.1074 & 0.2348 \\
\hline 3 & 0.0164 & -0.0013 & 0.2414 \\
\hline 4 & 0.0750 & -0.0440 & 0.2138 \\
\hline 5 & 0.0512 & -0.0011 & 0.2345 \\
\hline 6 & 0.0793 & -0.0186 & 0.2223 \\
\hline
\end{tabular}

$\delta_{\mathrm{cp}}^{2}=0.2310$

In the second row of selection

$$
b_{01}^{n}=0.0921, \quad b_{11}^{n}=-6.1055, \delta^{2}=0.195
$$

At the third stage, the model is obtained $\overline{\bar{y}}=0.0558+0.0213 \mathrm{X}_{2} \mathrm{X}_{3}+0.0085 \mathrm{X}_{3} \mathrm{X}_{4}-0.005 \mathrm{X}_{2} \mathrm{X}_{3}^{2} \mathrm{X}_{4}$

And so the object identification model

$\mathrm{y}=1.549-0.4614 \mathrm{X}_{1}+0.069 \mathrm{X}_{2}-0.5224 \mathrm{X}_{3}+0.063 \mathrm{X}_{4}-0.0617 \mathrm{X}_{1}^{2}+0.014 \mathrm{X}_{2}^{2}-$ $0.0002 \mathrm{X}_{4}^{2}+0.0213 \mathrm{X}_{2} \mathrm{X}_{3}+0.0085 \mathrm{X}_{3} \mathrm{X}_{4}-0.005 \mathrm{X}_{2} \mathrm{X}_{3}^{2} \mathrm{X}_{4}$. 


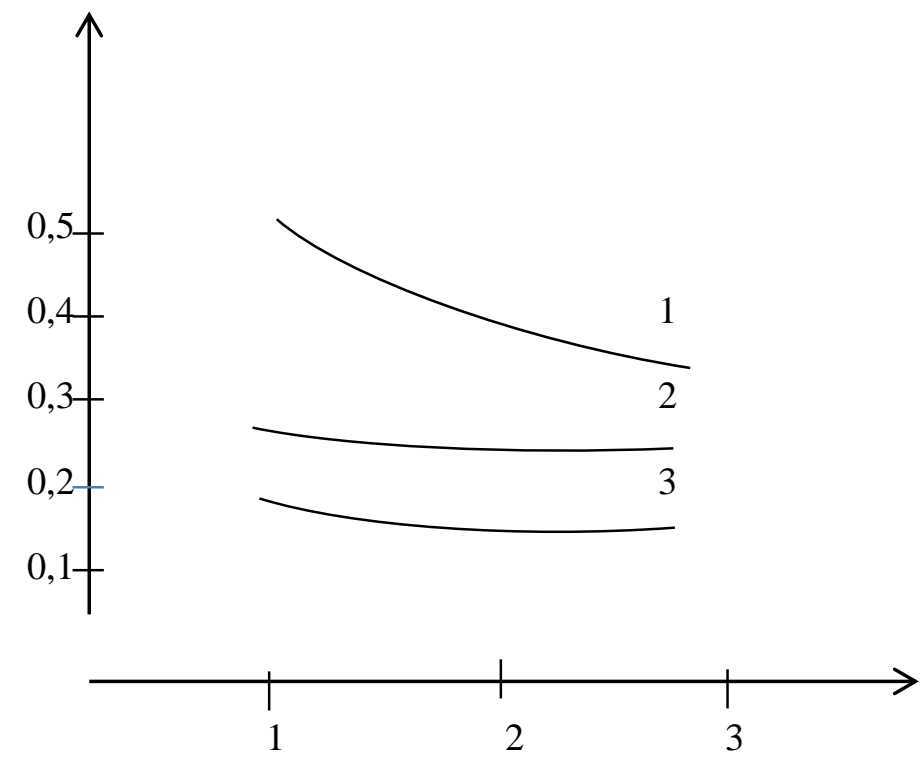

S

Fig. 1. Root mean square error from the number of breeding rows

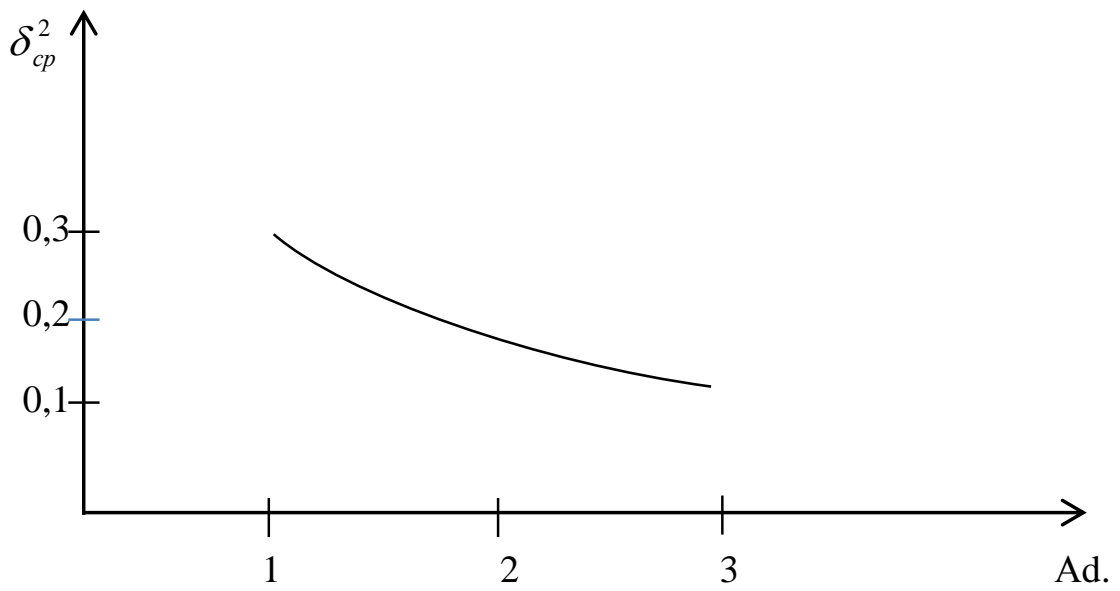

Fig. 2. Root mean square error on the number of process additivity

The dependence of the mean square error on the number of selection series in the first (curve 1), second (curve 2), and third stages (curve 3) are shown in Fig. 1, and the dependence of the mean square error on the additivity number is shown in Fig. 2. Thus, a methodology was developed for constructing nonlinear multipara meter mathematical models and was used in modeling and identifying parameters of the process of a supersonic flow around an oscillating profile.

\section{REFERENCE}

[1] Ivakhnenko A.G., Zaichenko R.G., Dimitrov V.D. Decision making on the basis of selforganization. M., Sov. Radio, 1976.280 p. 
[2] Boguslovsky I.A., Kruglyanova O.V., Miroshichev N.Ya., Tsymbalyuk G.M. Algorithm for identifying the parameters of a dynamic system of an aircraft. M. Izvestiya RAN. Theory and control systems 2009, №3, p. 3-15

[3] Safarov R. Additive self-organization algorithm. Scientific Journal of Samarkand State University. Exact sciences series. №3(115), 2019, c. 4-8. 